\title{
Jean Malaquais, une étoile filante de la littérature
}

\section{Henri Godard}

\section{(2) OpenEdition \\ Journals}

\section{Édition électronique}

URL : http://journals.openedition.org/rief/1295

DOI : 10.4000/rief.1295

ISSN : 2240-7456

\section{Éditeur}

Seminario di filologia francese

\section{Référence électronique}

Henri Godard, « Jean Malaquais, une étoile filante de la littérature », Revue italienne d'études françaises [En ligne], 6 | 2016, mis en ligne le 16 décembre 2016, consulté le 22 avril 2019. URL : http:// journals.openedition.org/rief/1295; DOI : 10.4000/rief.1295

Ce document a été généré automatiquement le 22 avril 2019.

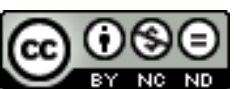

Les contenus de la RIEF sont mis à disposition selon les termes de la Licence Creative Commons Attribution - Pas d'Utilisation Commerciale - Pas de Modification 4.0 International. 


\title{
Jean Malaquais, une étoile filante de la littérature
}

\author{
Henri Godard
}

Les écrivains nés durant la décennie 1894-1904, c'est-à-dire ceux qui furent confrontés à la guerre, soit par une participation directe soit indirectement pendant leur adolescence, constituèrent en France une grande génération. Parmi les causes auxquelles on peut attribuer cette richesse, il y a le traumatisme de la guerre elle-même, mais aussi les bouleversements de tous ordres, politiques, sociaux, moraux et intellectuels qui s'en étaient suivis: multitude de questions nouvelles qu'il était naturel que la littérature s'emploie à prendre en charge, puisque c'est l'une de ses vocations. La guerre, par le nombre des tués et la présence parmi les survivants des blessés et des mutilés, avait fait de la mort et des questions existentielles qu'elle pose une réalité plus proche et plus dérangeante qu'elle ne l'est dans l'ordinaire des temps. Les mœurs et les rapports entre classes sociales n'étaient plus ce qu'ils étaient (le monde décrit par Proust semblait dater d'avant le déluge). Dans l'interprétation de l'expérience, freudisme et surréalisme mettaient en question la psychologie reçue et même le rationalisme. Spengler avait annoncé en 1918 le déclin de l'Occident, et, dans le même temps, le rapport de l'Europe, et de la France en particulier, avec les populations des autres continents ne pouvait rester ce qu'il était auparavant. Tout contribuait à ce changement: la participation de troupes coloniales au conflit, la prise de conscience chez quelques-uns du caractère insoutenable de la colonisation elle-même, l'attrait grandissant que les arts traditionnels de ces populations inspiraient aux artistes européens. Bientôt, dans les années 1920, allaient en outre faire leur apparition deux types nouveaux d'organisation sociale, communisme et fascisme puis nazisme, également étrangers à l'opposition française de la monarchie ou de l'empire et de la république, et qui exigeaient de chacun, écrivains compris, une prise de parti si ce n'est un engagement. Autant de sujets nouveaux par rapport à l'avantguerre, qui en France s'offraient à quiconque, habité par la littérature et désireux d'en prolonger la tradition dans sa langue, était sorti vivant de cette guerre. Dans ce domaine de la création, intervenaient qui plus est deux aspects nouveaux spécifiques. L'un était langagier. Le brassage social des combattants avait favorisé, dans ce français si 
étroitement cantonné depuis des siècles à une langue bourgeoise seule reconnue comme «bonne » et digne d'être écrite, l'émergence d'une langue populaire dans un usage de plus en plus large et qui devait finir par s'étendre à l'écrit. L'autre était la prise de conscience, accélérée par toutes les nouveautés contemporaines, d'une usure du modèle romanesque qui au XIX siècle, avec Balzac, Stendhal, Flaubert et Zola, avait fourni le moule de tant de chefs-d'œuvre, mais dont le roman russe de la fin du siècle avait révélé qu'il n'était pas le seul possible.

2 J'ai donné ce titre de Grande génération à un recueil d'articles consacrés à ceux de ces écrivains qui nous apparaissent aujourd'hui dans cette galaxie comme des étoiles de première grandeur, c'est-à-dire qui restent pleinement présents pour nous et sont nos références : Céline, Malraux, Giono, Aragon, Queneau ou Sartre. Mais cette génération est grande aussi par le nombre exceptionnel d'écrivains de valeur qui, du fait de cette richesse, se trouvent à l'heure actuelle sinon oubliés du moins relégués au second plan. À défaut d'être dans toutes les mémoires, ils ont des fidèles, lecteurs, critiques, éditeurs, pour qui ils restent vivants et qui tentent de faire partager ce sentiment, ou en tout cas de les inscrire dans l'histoire de la littérature. En témoigne à son tour notre travail ainsi que les sociétés d'Amis de tel ou tel parmi eux, et les republications de leurs œuvres. Leur nombre ne se limite pas à ceux qui sont évoqués ici. Parmi les autres, au premier rang desquels je mettrais personnellement Louis Guilloux, l'auteur du Sang noir, il faudrait citer aussi Henri Calet, Paul Nizan, Henri Thomas, Jean Prévost, Wladimir Pozner, Jacques Chardonne, Marcel Arland, Joseph Delteil, Philippe Soupault, Panait Istrati etc. Et ne faudrait-il pas compter dans leur nombre d'autres, qui figurent bien dans les Histoires de la littérature mais sont oubliés en ce sens qu'ils n'ont guère de présence vivante parmi les lecteurs? La métamorphose, dont Malraux a fait une loi de la destinée de toute œuvre, s'exerce parfois sous nos yeux : des auteurs dont à un moment donné l'audience et les chances de survie ont été, il y trente ou quarante ans, jugés assez grandes pour qu'on les inscrive au catalogue de la Bibliothèque de la Pléiade n'y figurent plus guère aujourd'hui, s'il faut en croire les chiffres de vente, qu'au titre de l'histoire de la littérature : Mauriac, Green, Montherlant par exemple.

3 Ceux qui font l'objet de cette étude sont dans une sorte de limbes, entre intérêt vivant et classement historique. Il revient à chacun de nous de dégager la part ou les aspects de leur œuvre qui suscitent individuellement en nous cet intérêt. C'est ce que je me propose de faire sur l'exemple de Jean Malaquais (1908-1998).

Malaquais est l'auteur de trois romans publiés et d'un quatrième resté inachevé, d'un recueil de nouvelles, de deux volumes de journal et d'une pièce de théâtre. Le premier roman, Les Javanais, faisait en 1939 une ouverture magistrale à l'œuvre qui aurait pu suivre. Je tentais en 2000 d'en mettre en valeur les mérites dans une étude intitulée «Les Javanais de Jean Malaquais, roman des sans-papiers et de leurs langues natales $»^{1}$ dont je me contenterai de rappeler l'essentiel pour m'intéresser à la production postérieure de Malaquais et à sa destinée.

Les Javanais est l'histoire, dans les années qui précèdent immédiatement la guerre de 1939, d'une communauté de mineurs apatrides venus d'une quinzaine de pays d'Europe et du pourtour de la Méditerranée travailler dans une mine d'argent et de plomb située en Provence. Ils lui ont donné le nom d'île de Java, à la fois par référence dérisoire au rêve de paradis exotique qui leur a fait fuir leur pays d'origine, et à cause du sabir dans lequel ils communiquent entre eux, et qui est inintelligible à qui n'appartient pas à leur communauté : le « javanais » est une forme traditionnelle d'argot fait pour n'être compris 
que des initiés. Ces "Javanais" sont en situation irrégulière et, à l'approche de la déclaration de guerre, sur le point d'être victimes d'un lock-out, mais ils se défendent et finissent par obtenir des compensations appréciables à leur renvoi.

6 Sur leur nombre, une douzaine est individualisée, mais aucun n'est privilégié par rapport aux autres par la narration. Grâce à une fragmentation et à un enchaînement rapide des discours intérieurs, le roman est l'histoire de leur être collectif fondu, malgré l'extrême diversité de leur origine, par leur identité d'exilés apatrides et leur condition d'ouvriers exploités. Avec un tel sujet, le roman a naturellement un premier niveau de dénonciation sociale, mais, pour plusieurs raisons, il ne s'y limite pas. La dénonciation n'est pas comme elle pourrait l'être empreinte d'indignation. Le ton qui domine est celui d'une satire non dépourvue de gaieté. Les Javanais ne perdent pas le sentiment que, exploités, ils ont au moins l'avantage d'être en-dehors du système social, de ses contraintes et de ses aliénations. Leur lutte, tout en étant âpre, est exaltante. Leur licenciement final ne sera pas sans leur donner un sentiment de libération. Chez eux, dans le même temps, la solidarité se double d'un sens de la fête. Trois personnages qui sont des exilés politiques d'origine sociale plus favorisée que les autres dotent enfin le récit d'une profondeur supplémentaire par des interrogations de nature existentielle. Malaquais fait partie de cette génération d'écrivains français pour qui protestation contre l'état social du monde et inquiétude philosophique vont de pair.

7 Sur le plan de la narration, le roman tire sa force de son modernisme qui prend la forme d'un morcellement qui brouille la chronologie et se garde d'un excès de rationalité dans la mise en évidence des causes et des conséquences (évitant par là la dramatisation trop facile d'une action sociale). Dans l'écriture, Malaquais, qui est un lecteur de Céline, a recours dans le discours intérieur des personnages à une langue orale populaire. Vu la diversité d'origine des Javanais, il obtient en outre un effet de plurivocalisme non seulement de voix mais de langues qui, au-delà de sa dimension stylistique, prend en l'occurrence une dimension humaine symbolique du fait qu'ils arrivent à se comprendre.

8 Trotsky, à l'époque, n'hésitait pas à intituler un compte rendu critique du roman « Un nouveau grand écrivain » comme il avait écrit six ans plus tôt à propos de Voyage au bout de la nuit : « L.F. Céline est entré dans la grande littérature comme d'autres pénètrent dans leur propre maison $»^{2}$. Mais ce qui se révéla vrai pour l'un ne se vérifia pas pour l'autre, malgré un prix littéraire qui était un début de reconnaissance. Survient la guerre puis la vie difficile en zone sud pour un juif apatride, à quoi s'ajoute la poursuite du visa qui lui permettra de fuir la France occupée et de gagner le continent américain où il passera presque tout le reste de sa vie. À partir de ce moment-là, sa production littéraire s'espace et peine à trouver sa voie.

9 Il avait publié avant-guerre, avec l'appui d'André Gide, quelques nouvelles en revue. Il en écrit alors deux autres pour en faire un volume publié à New York sous le titre Coups de barre. Le titre est significatif : par volonté de ne pas se répéter, Malaquais aborde des sujets nouveaux, au risque de ne pas donner au lecteur le sentiment d'un imaginaire unifié qui s'impose. Deux histoires sont situées sur des navires marchands. L'une met en scène le désir homosexuel et ses conflits au sein d'un équipage, l'autre tend vers le fantastique.

10 Publié en France en 1947, Planète sans visa aborde au contraire un sujet proche de celui des Javanais : à Marseille, pendant la guerre entre 1940 et 1942, réfugiés et apatrides sont non seulement sans papiers mais à la recherche d'un visa pour quitter la France, c'est-à-dire, dans leur situation, sauver leur vie. La sympathie du narrateur leur est aussi acquise 
qu'aux Javanais, et son antipathie à ceux qui s'opposent à eux. Cette identité de ton se double d'une identité de facture et de démarche narrative: discours intérieurs qui s'enchaînent sans présentation du locuteur, va-et-vient entre la langue écrite du narrateur et la langue familière populaire des personnages. Par rapport aux Javanais, le lecteur a un sentiment de déjà vu, comme s'il s'agissait d'un simple agrandissement du premier roman, sans certains des aspects de sa réussite. Avançant dans sa lecture, l'impression le gagne que cela pourrait se prolonger indéfiniment - et en effet le roman, qui avait six cents pages lors de sa publication, en a encore cinq cents dans la version révisée que nous lisons aujourd'hui. Il y a des lecteurs de Malaquais pour préférer cette orchestration amplifiée, mais pour les autres, le coup d'essai des Javanais reste le coup de maître.

L'accueil de Planète sans visa n'ayant pas été à la hauteur de l'attente de Malaquais, six ans plus tard il publie un livre tout différent, Le Gaffeur. Il s'agit moins d'un roman que d'un conte philosophique, aussi détaché de la réalité historique et sociale du moment que les deux premiers y étaient enracinés : dans une société - qui n'est située ni dans l'espace ni dans l'histoire - les esprits sont si totalement conditionnés par les organes dirigeants qu'à part un individu isolé (le narrateur) personne n'a conscience de ce conditionnement, si bien que, le dénonçant à tout bout de champ, il n'arrive qu'à faire figure de gaffeur. Le lecteur a trop vite compris l'intention pour pouvoir s'intéresser longtemps au récit.

À quarante-cinq ans, Malaquais cesse pratiquement d'écrire, mis à part de brefs efforts pour tenter sa chance dans d'autres genres, poèmes, pièces de théâtre et scénarios qui resteront inédits. En marge de ses œuvres de fiction ne seront publiés que deux volumes de Journal.

13 Ce dépérissement d'une production littéraire qui avait commencé par un coup d'éclat est en lui-même un sujet de réflexion. Une part en est certes imputable aux circonstances, l'exil et une vie matérielle toujours difficile, mais, dans cette destinée, il y a la question plus générale de savoir ce qui achève de faire, de quelqu'un qui a commencé à publier, l'auteur d'une œuvre au sens plein du terme.

Malaquais n'est pas un écrivain de vocation, ni même quelqu'un qui attache à la littérature une attention particulière. On ne le voit jamais, dans ses lettres et dans ses journaux, se référer à des admirations littéraires, de celles qui lui auraient donné envie de se consacrer à son tour à la littérature. On mesure la différence avec d'autres qui, eux non plus, n'ont pas eu un parcours traditionnel d'écrivain mais qui se sont, pour finir, identifiés à leur œuvre. Conrad, avec qui Malaquais avait en commun d'être Polonais, d'écrire dans une langue qui n'était pas sa langue maternelle, et d'avoir navigué pendant des années, passera à partir d'un certain point toute sa vie à écrire, non sans difficultés et déception au début, mais publiant au total assez de romans et de nouvelles pour faire, en traduction, la matière de quatre volumes de la Bibliothèque de la Pléiade. De son côté Céline pouvait bien soutenir, à l'époque de sa publication, qu'il n'avait écrit Voyage au bout de la nuit que pour payer son terme, cela ne l'empêcha pas, après ce premier succès, de continuer à écrire et de laisser une œuvre romanesque composée de huit romans.

L'écriture, selon Valéry, est une profession délirante. Il faut « croire » à la littérature pour juger naturel d'y sacrifier du temps et des forces aux dépens d'autres activités, ou seulement de la réflexion sur d'autres sujets. Toute sa vie, l'intérêt majeur de Malaquais a été la politique : le destin de la lutte à mener, aussi bien contre le capitalisme que contre le communisme soviétique, toujours dans une perspective d'internationalisme. Partout, ses fréquentations, ses échanges étaient dans cette mouvance. 

laisserais tomber la littérature et me plongerais à corps perdu dans le travail révolutionnaire $\rrbracket^{3}$. L'année suivante, il écrit à André Gide, son ami depuis les années 1930 : «Je suis fatigué d'écrire des histoires; je me sens coupable de vouer mes années à faire de la littérature quand il me faudrait formuler une pensée politique $»^{4}$. À être traitée ainsi, la littérature finit par se venger.

Il est toutefois à noter que, dans les trois dernières années de sa vie, à presque quatrevingt-dix ans, Malaquais revient finalement à celle-ci pour une révision stylistique attentive de ses deux premiers romans, comme s'il avait pris conscience in extremis que la création littéraire valait la peine qu'on porte chaque livre à son plus haut degré possible de réussite. À défaut de s'inscrire durablement dans le ciel de la littérature, Malaquais y aura au moins été une étoile filante.

\section{NOTES}

1. H. Godard, "Les Javanais de Jean Malaquais, roman des sans-papiers et de leurs langues natales ", dans Littératures contemporaines, 8, 2000 : Quinze romans dans le siècle, études réunies par A. Cresciucci, p. 115-126.

2. Ces deux comptes rendus figurent dans le recueil Littérature et révolution: L. Trotsky, «Un nouveau grand écrivain ", dans Littérature et révolution, préface de M. Nadeau, traduit du russe par P. Frank, C. Ligny et J.-J. Marie, Paris, Les Éditions de la Passion, 2000, p. 192-198 ; L. Trotsky, «Céline et Poincaré ", ibid., p. 233-240, p. 233.

3. Cité par G. Nakach, Malaquais rebelle, Paris, Éditions du Cherche Midi, 2011, p. 253.

4. A. Gide, J. Malaquais, Correspondance (1935-1950), Paris, Éditions Phébus, 2000, p. 207.

\section{RÉSUMÉS}

Au sein de la «Grande génération » des écrivains nés entre 1894 et 1904, que les bouleversements produits par la guerre avaient amenés à prendre conscience du déclin du modèle romanesque du $\mathrm{XIX}^{\mathrm{e}}$ siècle, cet article attire l'attention sur Jean Malaquais, et notamment sur son roman le plus représentatif, Les Javanais (1939). Dans cet ouvrage les éléments de la dénonciation sociale et de la problématique existentielle sont appuyés, sous l'aspect formel, par l'emploi de la langue orale populaire, du plurivocalisme et du discours intérieur. Sa production réduite et sa considération même de l'écriture comme secondaire par rapport à l'engagement politique actif, font de Malaquais une « étoile filante » de la littérature. 
INDEX

Mots-clés : Oubliés, années Trente, engagement, Malaquais (Jean), Les Javanais 